\title{
Ring finger protein 6 promotes breast cancer cell proliferation by stabilizing estrogen receptor alpha
}

\author{
Yuanying Zeng ${ }^{1,2, *}$, Xin Xu ${ }^{1,3, *}$, Siyu Wang ${ }^{1, *}$, Zubin Zhang ${ }^{1}$, Yan Liu ${ }^{1}$, Kunkun Han ${ }^{1}$, \\ Biyin $\mathrm{CaO}^{1}$, Xinliang Mao, ${ }^{1,4,5}$ \\ ${ }^{1}$ Jiangsu Key Laboratory for Translational Research and Therapeutics of Neuro-Psycho- Diseases, Department of Pharmacology, \\ College of Pharmaceutical Sciences, Soochow University, Suzhou, Jiangsu, 215123, P. R. China \\ ${ }^{2}$ Department of Oncology, Suzhou Municipal Hospital East Campus, Suzhou, 215100, P.R. China \\ ${ }^{3}$ Suzhou Institute of Systems Medicine, Center of Systems Medicine, Chinese Academy of Medical Sciences, Suzhou, 215123, \\ P. R. China \\ ${ }^{4}$ Jiangsu Key Laboratory of Preventive and Translational Medicine for Geriatric Diseases, Soochow University, Suzhou, \\ 215123, P. R. China \\ ${ }^{5}$ Key Laboratory of Protein Modification and Degradation, School of Basic Medical Sciences, Affiliated Cancer Hospital \& \\ Institute of Guangzhou Medical University, Guangzhou 511436, P. R. China \\ "These authors have contributed equally to this work \\ Correspondence to: Yuanying Zeng, email: zengyuanying@163.com \\ Xinliang Mao, email: xinliangmao@suda.edu.cn
}

Keywords: ring finger protein 6, breast cancer, $E R \alpha, B C l-x L$, doxorubicin

Received: November 30, 2016

Accepted: January 22, 2017

Published: February 16, 2017

\section{ABSTRACT}

Ring finger protein 6 (RNF6) is a key oncogene in both prostate cancer and leukemia, but its role is elusive in breast cancer. In the present study, we found that RNF6 was overexpressed in more than $70 \%$ of breast cancer tissues and it was associated with overall survival. RNF6 increased breast cancer cell proliferation, migration and reduced cell sensitivity to doxorubicin. Further studies showed that RNF6 was closely associated with increased expression of estrogen receptor, a critical factor in the development of breast cancers. RNF6 was found to induce ER $\alpha$ expression and increased its stability. In doxorubicin-resistant breast cancer cells, RNF6 was found to be elevated in association with increased ER $\alpha$ and anti-apoptotic $B c l-x L$, but not pro-apoptotic Bim-1. In consistence with this finding, overexpression of ER $\alpha$ led to increased $\mathrm{BCl}-\mathrm{xL}$ but had no effects on Bim-1. Therefore, this study demonstrated that there exists an RNF6/ER $\alpha / B c l-x L$ axle in breast cancer which promotes cancer cell proliferation and survival. Targeting the RNF6/ER $\alpha / B c l-x L$ axle could be a promising strategy in the treatment of breast cancer.

\section{INTRODUCTION}

Breast cancer is one of the most common forms of cancers in women. According to the information from World Cancer Report, It was estimated that there were 1.7 million new cases and 0.5 million cancer deaths in 2012 worldwide, which both ranked the highest among all female cancers [1]. The National Cancer Registry of China also reported that breast cancer has emerged as the most registered cancer in women in China. The average incidence of breast cancer in women has reached 42.55 per 100,000 and it increases with age [2]. The diagnosis and therapeutic advances have improved the survival of breast cancer patients with early stages, but some patients become resistant and refractory even with optimized therapeutic methods. Genetic complexity has been proposed as a key factor in breast cancer treatment and predictive outcomes.

The ring finger protein 6 (RNF6) is a ubiquitin ligase that was originally cloned in a genetic study of chromosomal rearrangements in myeloproliferative disorders [3]. Because of its mapping to chromosome $13 \mathrm{q} 12$ and mutations in human esophageal squamous cell cancers, RNF6 was originally believed as a tumor suppressor gene [4]. However, RNF6 was found to mediate an atypical ubiquitination of the androgen 
receptor $(\mathrm{AR})$ and promotes the transcriptional activity and specificity of AR in prostate cancer [5]. Therefore, RNF6 is proposed as an oncogene in the development and progression of prostate cancers and it is required for prostate cancer growth [5]. Recently we found that RNF6 is overexpressed in hematological cancers, including leukemia, lymphomas and multiple myeloma as a direct gene of the transcription factor Pbx1 [6]. RNF6 induces the proliferation of leukemia cells, whereas knockdown of RNF6 delayed the growth of tumors derived from human leukemia cell line K562 in mice [6]. Therefore, RNF6 has been demonstrated as an oncogene, but its specific roles in breast cancers are not yet understood.

The present study found that RNF6 is overexpressed in more $70 \%$ of breast cancer tissues in comparison with individual para-cancerous tissues. RNF6 increases the stability of estrogen receptor alpha (ER $\alpha)$, a key player in the pathophysiology of breast cancers. Targeting the RNF6/ER $\alpha$ axle could be a promising strategy for the treatment of breast cancers.

\section{RESULTS}

\section{RNF6 is overexpressed in various breast cancer tissues and breast cancer cell lines}

RNF6 has been found as an oncogene that is highly expressed in prostate cancer and leukemia $[5,6]$. To find out its expression profile in breast cancer, a panel of breast cancer tissues and their individual para-cancerous tissues were collected for RNF6 expression by qRT-PCR. As shown in Figure 1A, RNF6 was found to be upregulated in more than $70 \%(20 / 27)$ of representative primary breast cancer tissues compared with their para-cancerous control tissues. This finding was confirmed by RT-PCR as shown in Figure 1B. To further confirm the protein level of RNF6 in human breast cancer, 136 infitrating ductal carcinoma tissues were collected and tissue arrays were performed by immunohistochemical (IHC) staining with a specific RNF6 antibody. As shown in Figures 1C and 1D, RNF6 was highly expressed in human breast cancer compared with their para-cancerous tissues. All the results clearly showed that RNF6 was dysregulated in breast cancer tissues. To find out whether RNF6 was also expressed in breast cancer cell lines, MCF-7, MDA-231, MDA-453, and T47D were subjected to immunoblotting for RNF6 protein expression. As shown in Figure 1E, all cell lines examined displayed a high level of RNF6, in contrast, it was unable to be detected in a normal breast tissue cell line MCF-10A. This finding was consistent to previous reports that RNF6 was dysregulated in cancers such as leukemia [6] and prostate cancers [5]. Of interest, among these cell lines tested, T47D and MCF-7 are highly expressing estrogen receptor and MDA-MB-231 and MDA-MB-453 express relatively low levels of ER [7]. These results probably implicated that RNF6 was associated with ER expression.

\section{RNF6 predicts a poor prognosis of breast cancer patients}

RNF6 was highly expressed in both breast cancer tissues and cell lines, we wondered whether RNF6 was clinically important. To this end, we next evaluated the association of RNF6 expression and the survival period of breast cancer patients. Patients were divided into two groups based on RNF6 expression, RNF6-low ( $n=43$ ) and RNF6-high ( $\mathrm{n}=93)$. Survival rates of patients within 5 years (60 months) were calculated and compared using the Kaplan-Meier estimate [10]. The result indicated that RNF6 was associated with the survival period of breast cancer patients. As shown in Figure 2, > 95\% of breast cancer patients with a low expression of RNF6 survived more than 60 months, while $<80 \%$ patients expressing a high level of RNF6 survived over 60 months. This analysis suggested that RNF6 probably predicted a poor prognosis of breast cancer patients.

\section{RNF6 is associated with age, clinical stage, ER and $P R$ expression in patients with invasive ductal breast carcinoma}

The above studies had showed that RNF6 was highly expressed in breast cancer tissues and was associated with patient survival, to further measure the effects of RNF6 in primary patients, 136 breast cancer tissues were applied for clinical evaluation in terms of patients' age, clinical stage, the expression of RNF6, ER $\alpha$, PR and HER2, because the trio of ER $\alpha$, PR and HER2 are proposed indicators for the prognosis of breast cancer patients. The Chi square analyses revealed that RNF6 expression was significantly associated with age. Patients $>50$ years old expressed a higher level of RNF6 than younger ones. In addition, RNF6 was associated with clinical stages and the expression of ER $\alpha$ and PR but not HER2 (Table 1). Compared with patients with negative ER $\alpha$ and/or PR expression, a high level of RNF6 was detected in patients with positive $\operatorname{ER} \alpha(p=0.018)$ and $\operatorname{PR}(p=0.002$, Table 1 ). Therefore, we hypothesized that RNF6 was probably associated with the expression of ER $\alpha$ and PR.

\section{RNF6 promotes proliferation and migration of breast cancer cells}

RNF6 was highly expressed in both breast cancer tissues and it was associated with poor prognosis, then we wondered whether RNF6 contributed to breast cancer cell proliferation. To this end, RNF6 plasmid was transfected into MCF-7 cells, followed by cell proliferation assay using MTT assay. The result showed that RNF6 promoted 
A

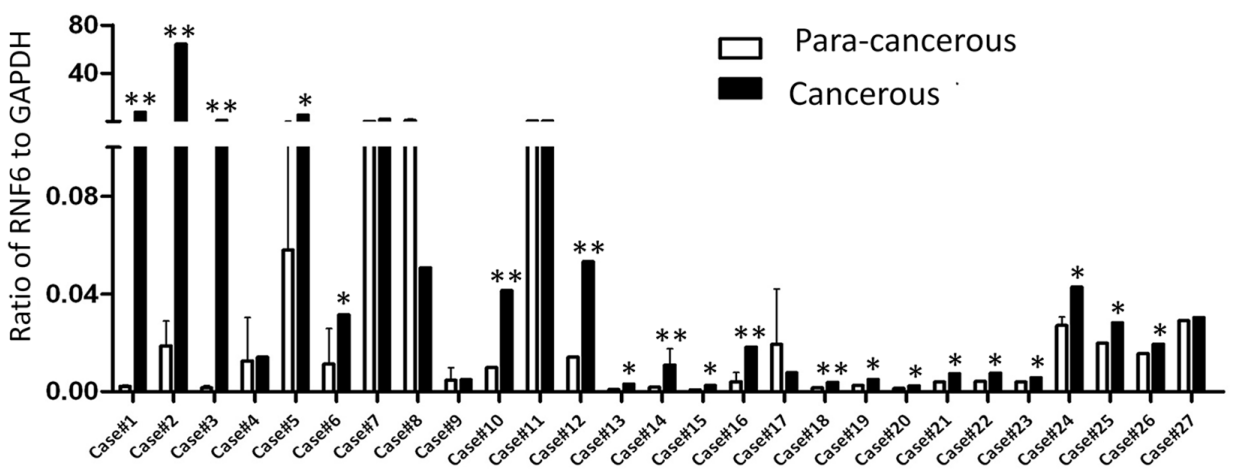

B

$\# 1 \quad \# 2 \quad \# 3 \quad \# 6 \quad \# 24 \quad \# 25$

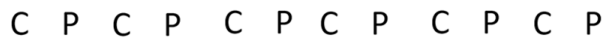

RNF6
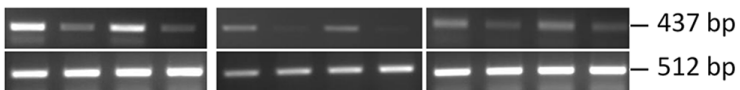

GAPDH $-\infty$

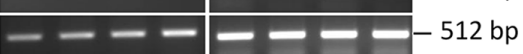

D

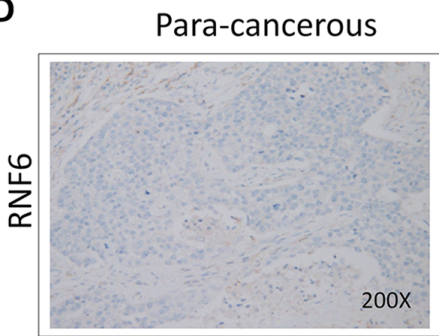

Cancerous

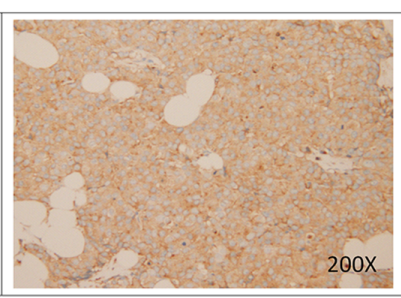

C

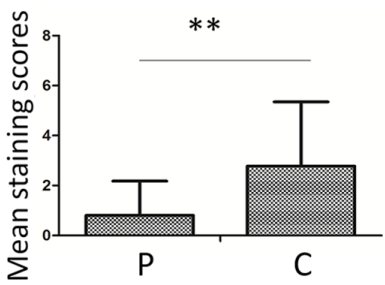

E

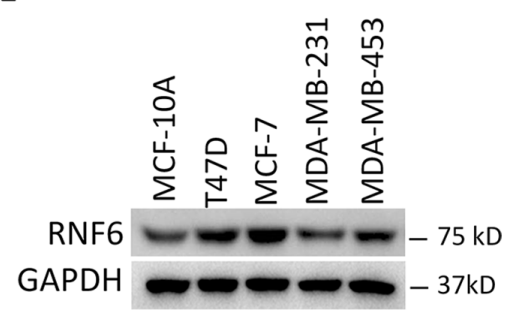

Figure 1: RNF6 is overexpressed in breast cancer. A. Fresh primary breast cancer tissues and individual para-cancerous tissues were analyzed for RNF6 expression by qRT-PCR. B. RNF6 was detected by RT-PCR using tissues from representative patients as listed in A. C: Cancer tissues; P: para-cancerous tissues. C. Statistical analysis of human breast cancer tissue arrays ( $\mathrm{n}=136)$ stained with an anti-RNF6 antibody. Immunostaining scores (mean \pm SD) for RNF6 in para-cancerous (P) and cancerous (C) tissues were summarized. D. Representative fields of human breast cancer tissue arrays by immunohistochemical staining using an anti-RNF6 antibody. E. The wholecell lysates from a normal breast cell line and various breast cancer cell lines were extracted, followed by detecting RNF6 protein levels measured by immunoblotting analyses. GAPDH was used as a loading control.* $p<0.05,{ }^{* *} p<0.01$.

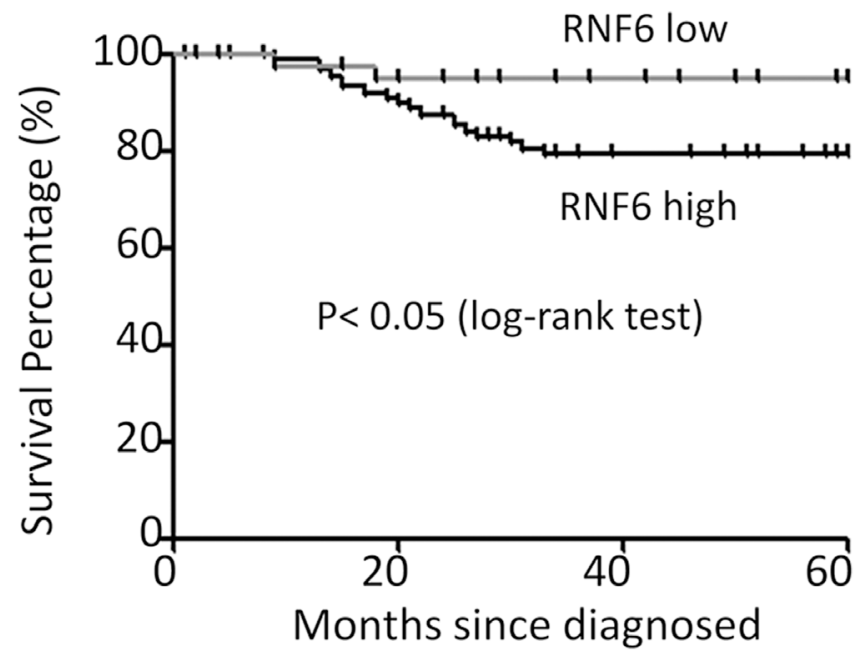

Figure 2: RNF6 is a negative index for the survival of breast cancer patients. The survival periods of breast cancer patients were estimated using the Kaplan-Meier estimates as described previously (see Methods). All patients were classified into two groups based on the RNF6 expression level. Estimated survival percentage of each group of patients was calculated. 
Table 1: RNF6 is associated with patient age, clinical stage, ER and PR expression in breast cancer tissues

\begin{tabular}{|c|c|c|c|c|}
\hline \multirow{2}{*}{ Variables } & \multicolumn{3}{|c|}{ RNF6 Staining } & \multirow{2}{*}{$P$ value* } \\
\hline & High & Low & Total & \\
\hline \multicolumn{5}{|l|}{ Age (years) } \\
\hline$<50$ & 25 & 19 & 44 & \multirow{2}{*}{0.045} \\
\hline$\geq 50$ & 68 & 24 & 92 & \\
\hline \multicolumn{5}{|c|}{ Lymph node metastasis } \\
\hline Negative & 64 & 31 & 95 & \multirow{2}{*}{0.502} \\
\hline Positive & 30 & 11 & 41 & \\
\hline \multicolumn{5}{|c|}{ Histology grade } \\
\hline 1 & 0 & 1 & 1 & \multirow{3}{*}{0.402} \\
\hline 2 & 42 & 18 & 60 & \\
\hline 3 & 51 & 24 & 76 & \\
\hline \multicolumn{5}{|c|}{ Clinical stage } \\
\hline I-II & 62 & 47 & 109 & \multirow{2}{*}{0.019} \\
\hline III-IV & 22 & 5 & 27 & \\
\hline \multicolumn{5}{|l|}{ ER } \\
\hline Negative & 9 & 12 & 21 & \multirow{2}{*}{0.018} \\
\hline Positive & 80 & 35 & 115 & \\
\hline \multicolumn{5}{|l|}{$\mathbf{P R}$} \\
\hline Negative & 7 & 13 & 20 & \multirow{2}{*}{0.002} \\
\hline Positive & 82 & 34 & 116 & \\
\hline \multicolumn{5}{|l|}{ HER2 } \\
\hline Negative & 19 & 12 & 31 & \multirow{2}{*}{0.237} \\
\hline Positive & 76 & 29 & 105 & \\
\hline
\end{tabular}

* $P$ value was calculated by using the $C h i$ square $\left(\chi^{2}\right)$ analysis based on RNF6 expression levels and patient numbers in each group.

MCF-7 proliferation in a time-dependent manner (Figure 3A). Because RNF6 was overexpressed in MCF-7 cells, we next knocked down RNF6 in these cells by lentiviral shRNA (shRNF6) followed by cell proliferation assay. As shown in Figure 3B, RNF6 was markedly downregulated by shRNF6 which attenuated breast cancer cell proliferation.

Increased cell migration is critical for the malignancy of breast cancer, therefore, we next evaluated whether RNF6 contributed to such a feature in breast cancer cells. Using scratch wound healing assay, a widely accepted method to measure cell migration, we found that MCF-7 cells with transfected RNF6 displayed stronger healing ability than control cells (Figures $3 \mathrm{C}$ and 3D), suggesting that RNF6 might contribute to breast cancer cell migration.

\section{RNF6 increases breast cancer cell resistance to anti-cancer agents}

Chemoresistance is an obstacle to clinical scientists and oncologists in breast cancer treatment. Previous studies showed that RNF6 has been found to be associated with chemoresistance of prostate cancer [5], therefore we wondered whether RNF6 also contributed to drug insensitivity of breast cancer. To this end, MCF-7 cells were treated with doxorubicin (ADR) [8], a mainstay drug in breast cancer treatment, or 5-amino-8-hydroquinoline (5AHQ) [9], a potential anti-cancer agent, for $24 \mathrm{hrs,}$ followed by immunoblotting. The results showed that both ADR and 5AHQ could downregulate RNF6 expression in breast cancer cells in a concentration- and time-dependent manner (Figure 4A-4C). When transduced with a RNF6 
plasmid, MCF-7 cells became resistant to 5AHQ (Figure 4D) and ADR (Figure 4E). This finding was consistent with the above study that RNF6 promoted breast cancer cell proliferation, migration and chemoresistance.

\section{RNF6 upregulates the expression level of ERa}

The above histochemical tissue array studies suggested that RNF6 was associated with ER $\alpha$, an important gene in breast cancer pathophysiology. Because RNF6 could be downregulated by anti-agents, we wondered whether the agents could also decrease ER expression. To this end, the same blots from Figure 4A in which MCF-7 cells treated with ADR and $5 \mathrm{AHQ}$, respectively, were stripped and subjected to immunoblotting against ER $\alpha$. As shown in Figure 5A, ER $\alpha$ was downregulated by both agents, in a similar manner to the effects of RNF6 (Figure 4A). This results implicated that RNF6 probably modulates ER $\alpha$ expression.

Previous studies have demonstrated that RNF6 as a ubiquitin ligase stabilized AR protein in prostate cancer patients [5], therefore, we evaluated the effects of RNF6 on the protein expression of $\mathrm{ER} \alpha$. As shown in Figure 5B, RNF6 increased ER $\alpha$ in a concentration-dependent manner in HEK293 cells when co-transfection of RNF6 and ER $\alpha$. We next wondered whether RNF6 had any effects on the protein level of endogenous ER $\alpha$. To this end, MCF-7 cells were transfected with RNF6 followed by immunoblotting. As shown in Figure 5C, the endogenous expression level of ER $\alpha$ was also markedly induced by RNF6. The above findings were confirmed by measuring $\mathrm{ER} \alpha$ stability in the presence of RNF6 after protein synthesis was prevented by cycloheximide (CHX). As shown in Figure 5D and $5 \mathrm{E}, \mathrm{RNF} 6$ markedly prevented degradation of ER $\alpha$ and significantly extended the half life of ER $\alpha$.

\section{RNF6 stabilizes the $\mathbf{E R} \alpha / \mathrm{Bcl}-\mathrm{xL}$ axle in a ubiquitination-independent manner}

The above studies showed that RNF6 stablizes ER $\alpha$, to find out its significance in breast cancer, we analyzed several genes in ADR-resistant MCF-7 (MCF-7 ${ }^{\mathrm{R}}$ ) cells. As shown in Figure 6A, both RNF6 and ER $\alpha$ were raised in $\mathrm{MCF}-7^{\mathrm{R}}$ cells compared with the wild-type parental
A

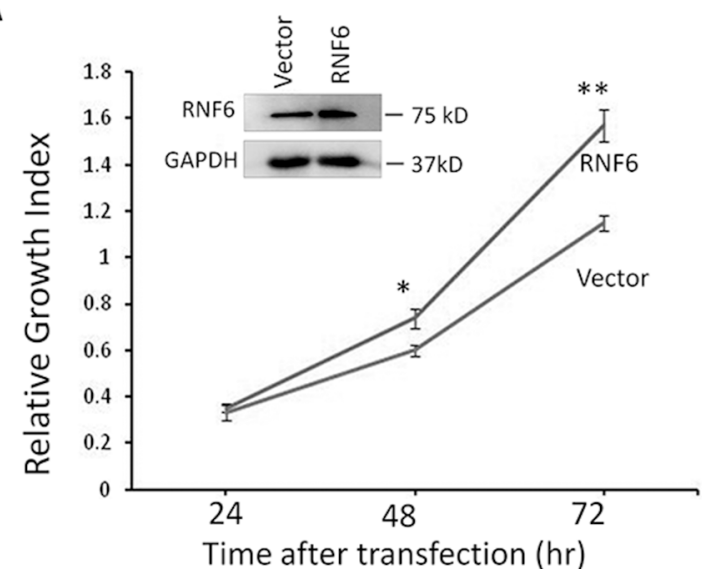

C

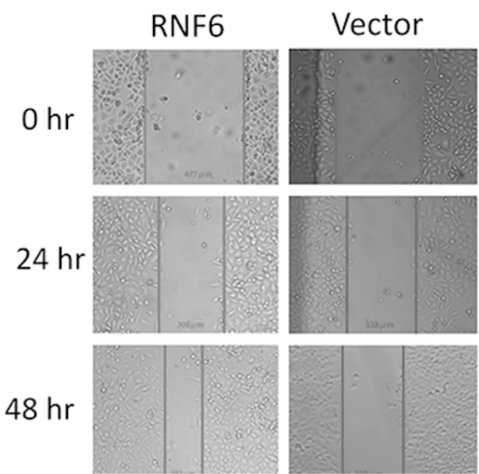

B

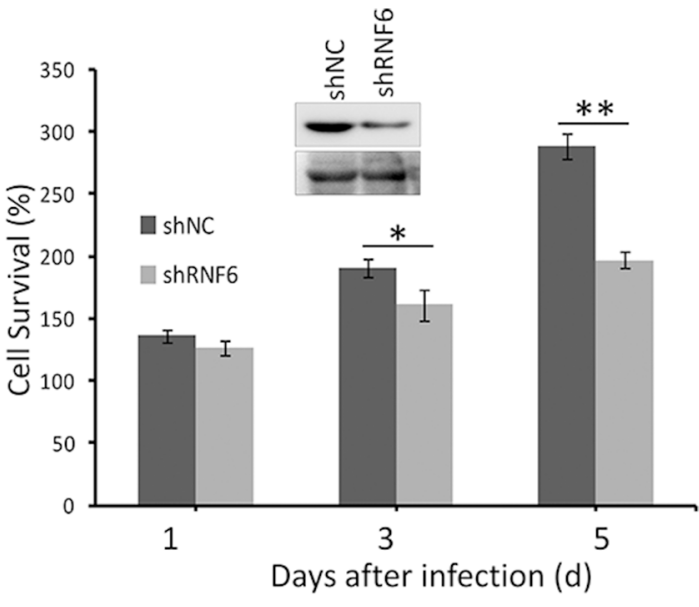

D

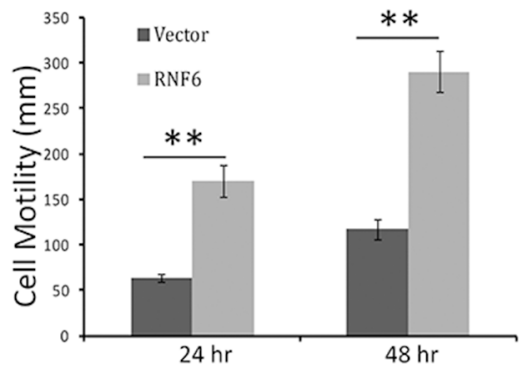

Figure 3: RNF6 promotes breast cancer cell proliferation in MCF-7 cells. A. MCF-7 cells were transfected with a RNF6 plasmid or empty vector. Cells were then plated into 96-well plates and continued to culture for 24, 48 or 72 hrs before cell number counting. B. MCF-7 cells were infected with lentiviral shRNA of RNF6 (shRNF6) or negative control (shNC). Cell viability was evaluated at Day 1,3, and 5, respectively. C and D. RNF6-transfected or control MCF-7 cells were scratched with a tip, followed by culture in a $37^{\circ} \mathrm{C}$ incubator for 24 or $48 \mathrm{hrs}$. The wound gaps were analyzed by a microscope (C) followed by statistical analysis (D). 
MCF-7. Because MCF-7R is resistant to ADR [10], we wondered whether there were any changes of the prosurvival and pro-apoptotic proteins using Bcl-xL and Bim as a representative, respectively $[11,12]$. As shown in Figure 6A, Bcl-xL was upregulated while Bim-1 was downregulated in $\mathrm{MCF}-7^{\mathrm{R}}$ cells, which was consistent with previous reports and the sensitivity of $\mathrm{MCF}-7^{\mathrm{R}}$ cells to ADR. We subsequently wondered whether ER $\alpha$ could modulate the expression of Bcl-xL and Bim-1. To this end, MCF-7 cells were transfected with an ER $\alpha$ plasmid followed by immunoblotting. As shown in Figure 6B, Bcl-xL was increased by ER $\alpha$ but Bim-1 was not affected. Therefore, it could be concluded that RNF6 probably modulates the ER $\alpha / \mathrm{Bcl}-\mathrm{xL}$ axle in breast cancer cells.

RNF6 has been reported to be a ubiquitin ligase that mediates atypical ubiquitination of adrogen receptor and contributes to prostate cancer progression [5]. To find out whether RNF6 also excutes in a similar manner to stabilize ER $\alpha$, we made a mutant RNF6 construct $\left(\mathrm{RNF}^{\triangle \mathrm{RING}}\right)$ that lacked the RING domain which is critical

A

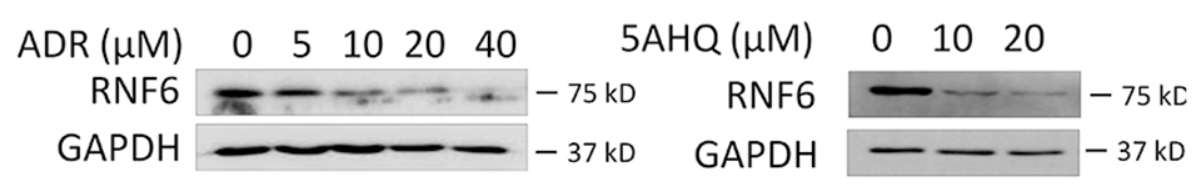

B

\section{C}
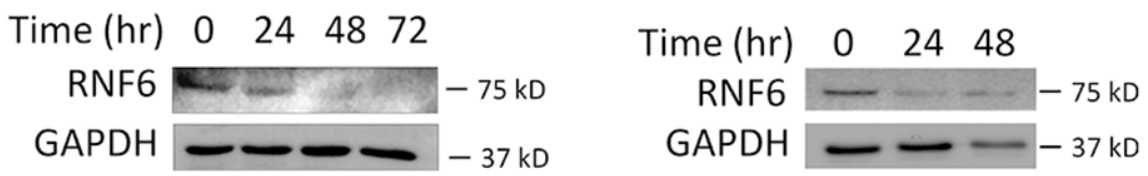

D

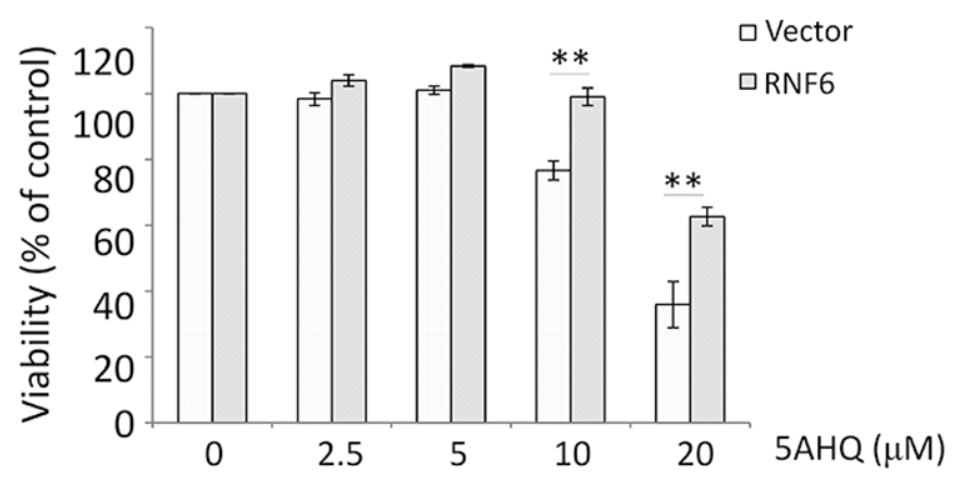

E

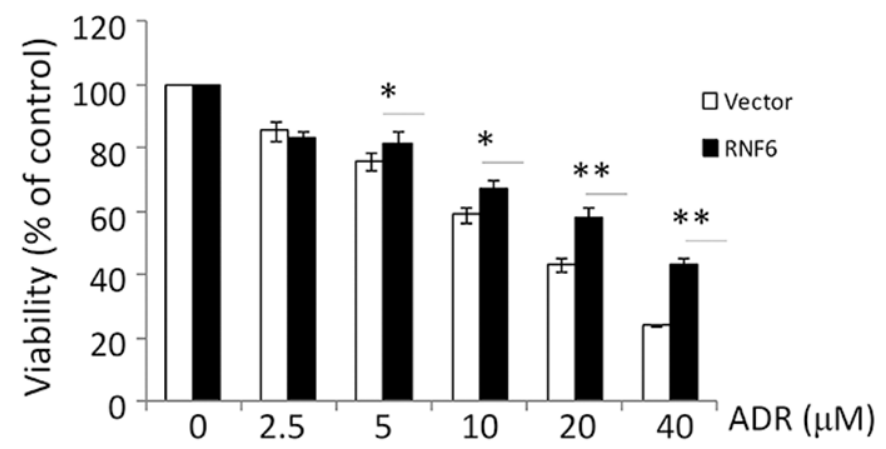

Figure 4: RNF6 increases MCF-7 resistance to anti-cancer agents. A. MCF-7 cells were treated with increasing concentrations of doxorubicin (ADR) or A5HQ for 24 hrs, followed by cell lysate preparation and immunoblotting against RNF6 or GAPDH. B and C. MCF-7 was treated with ADR $(16 \mathrm{M})$ or 5AHQ $(20 \mu \mathrm{M})$ for indicated time points, followed by cell lysate preparation and immunoblotting against RNF6 or GAPDH. D and E. MCF-7 cells transfected with RNF6 plasmid or empty vector (as shown in Figure 1A) for 24 hrs, followed by treatment with 5AHQ (D) or ADR (E) at indicated concentrations for 48 hrs. Cell viability was measured by MTT. 
A

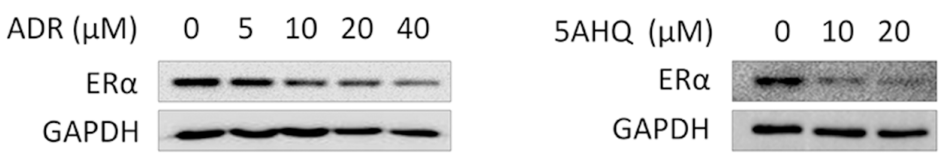

B

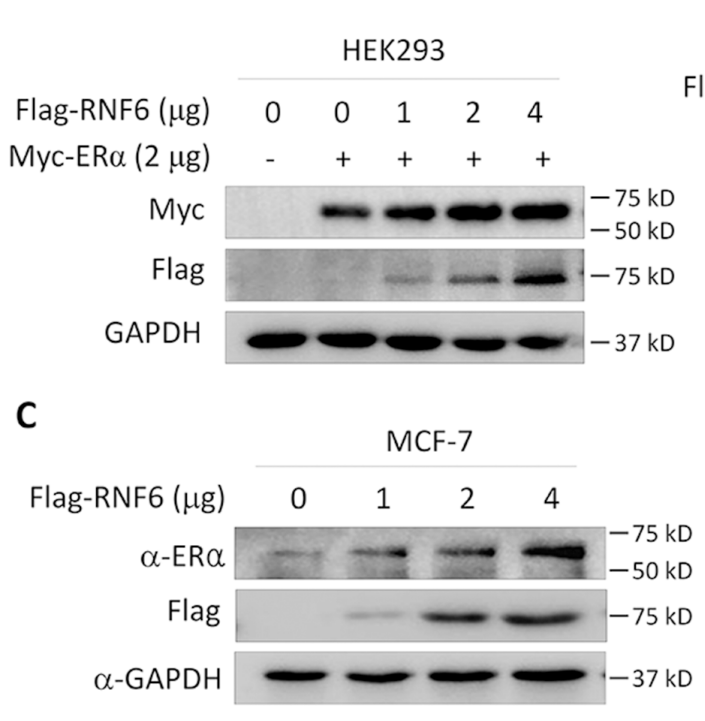

D

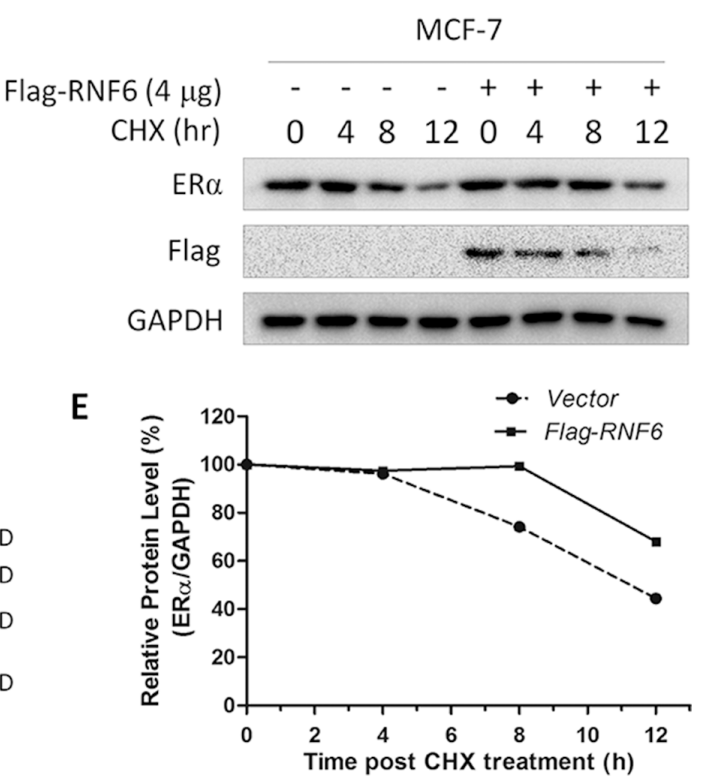

Figure 5: RNF6 increases the protein level of ERa. A. MCF-7 cells were treated with increased ADR or 5AHQ for 24 hrs, followed by cell lysate preparation and immunoblotting assay with specific antibodies as indicated. These blots were stripped from Figure 4A and then subjected to analysis for ER $\alpha$ and GAPDH. B. RNF6 and ER $\alpha$ were co-tranfected into HEK293 cells. Forty-eight hours later, cells were lysed for immunoblotting analysis. C. MCF-7 cells were transfected with increased RNF6 plasmids, followed by immunoblotting against ER $\alpha$, Flag and GAPDH. D. MCF-7 cells were transfected with Flag-RNF6 or vector for 24 hrs, followed by CHX chase assay. Immunoblotting analysis was performed against ER $\alpha$ and Flag. GAPDH was used as a loading control. E. Statistically analysis of Figure D.

A

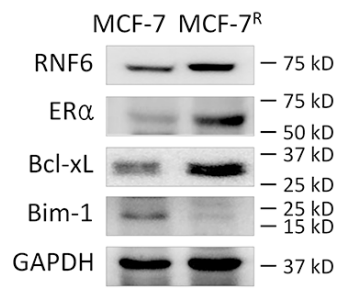

B

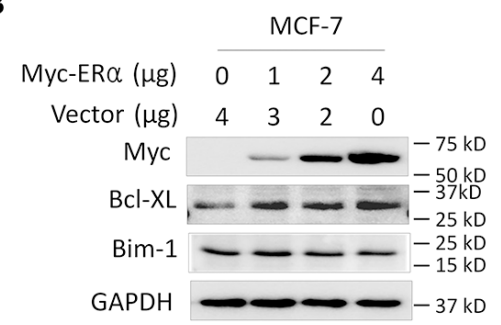

C

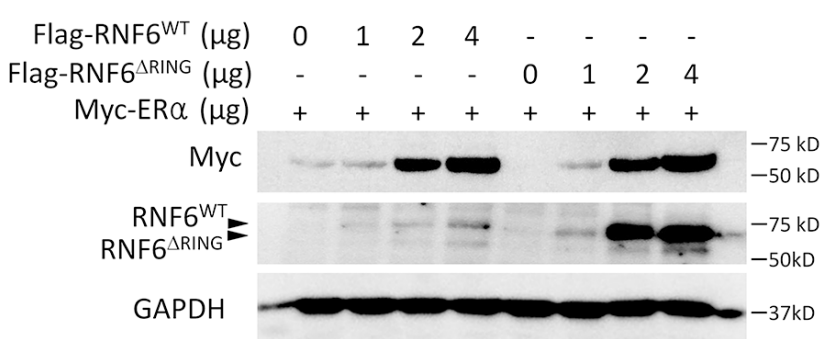

Figure 6: RNF6 promotes the ER $\boldsymbol{\alpha} / \mathbf{B c l}-\mathbf{x L}$ axle in breast cancer. A. ADR-resistant MCF-7 (MCF-7 ${ }^{\mathrm{R}}$ ) cells were measured associated gene expression. B. MCF-7 cells were transfected with increased Myc-ER $\alpha$ plasmids for 48 hrs, followed by immunoblotting analysis. C. Wild-type or RING-deficient RNF6 plasmids were co-transfected with Myc-ER $\alpha$ in HEK293 cells. Forty-eight hours later, cells were harvest for immunoblotting analysis against Myc and Flag. GAPDH was used as a loading control. 
for the ubiquitinating activity. After co-transfected with $\mathrm{ER} \alpha / \mathrm{RNF}^{\mathrm{ARING}}$ or ER $\alpha / \mathrm{RNF}^{\mathrm{WT}}$ cells were subject to immunoblotting. As shown in Figure $6 \mathrm{C}$, both the wildtype and RING-deleted mutant RNF6 could increase ER $\alpha$ protein which suggesting that RNF6 raised the ER $\alpha$ protein probably in a manner rather than protein ubiquitination because if RNF6 acted as a ubiquitin ligase, its mutant lacking the RING domain will not increase ER $\alpha$.

\section{DISCUSSION}

The above studies demonstrated that the ubiquitin ligase RNF6 was highly expressed in breast cancers and it probably promotes cell proliferation, migration and prosurvival of breast cancer cells by modulating the ER $\alpha / \mathrm{Bcl}-$ $\mathrm{xL}$ axle in a ubiquitination-independent manner.

RNF6 is first reported as a tumor suppressor gene not only because RNF6 is mapped to chromosome $13 \mathrm{q} 12$ [3], a location harboring several other suppressor genes such as p53 and PTEN, but also because its mutations are found in the gene of RNF6 in esophageal squamous cell carcinoma [4]. However, our present study demonstrated that RNF6 is oncogenic not only because of its high expression frequency in breast cancer tissues and cells, but because of its ability to increase breast cancer cell proliferation, migration and chemoresistance. This finding is consistent with its role in prostate cancer and leukemia $[5,6]$. RNF6 as an oncogene promotes prostate cancer progression [5]. Recently, we found that RNF6 is highly expressed in leukemia, myeloma, and other hematological malignancies and contributes to leukemia cell proliferation in vitro and in vivo [6]. The present study showed that RNF6 is dysregulated in more than $70 \%$ of breast cancer tissues and it is negatively associated with the survival period of breast cancer patients, which suggesting RNF6 is probably associated with its specific pathophysiological activity.

HER2, ER and PR are critical biomarkers in the classification of breast cancers, predicating clinical outcomes and reference to therapeutic strategies [13, 14]. Based on the expression of this trio, one of the group is identified as tri-negative breast cancer (TNBC) which represents around $15 \%$ of all breast cancers and is characterized by shorter overall survival [15]. Based on our study, RNF6 is found to be associated with increased expression levels of ER and PR but not HER2, therefore, compared with the TNBC, although the survival period in patients with $\mathrm{RNF}^{\text {low }}$ is markedly longer than those with RNF6 ${ }^{\text {high }}$, RNF6 and associated ER expression might predict a favorable outcome compared TNBC. However, the underlying mechanisms are not known and deserves further investigation.

RNF6 is involved in prostate cancer progression by mediating the K63-chain ubiquitination on androgen receptor thus modulating AR transcriptional activity and specificity [5]. Different from MDM2, another ubiquitin ligase, that mediates polyubiquitination and stability of AR, RNF6 seems to have no effects on AR stability [5]. Although both AR and ER are nuclear receptors, RNF6 can stabilize ER $\alpha$ protein which was clearly demonstrated by exogenous and endogenous ER $\alpha$ proteins in presence of RNF6. It is well known that the RING domain is critical for the ubiquitin ligase activity of RNF6 and the deletion of the RING domain will abolish the ubiquitinating ligase activity [5]. As shown in the AR study, RNF6 ${ }^{\triangle R I N G}$ fails to induce AR polyubiquitination, however, this mutant can increase ER $\alpha$ stability in a potency similar to the wild type (Figure 6). Actually, co-transfection of RNF6 ${ }^{\Delta \mathrm{RING}}$ leads to a raised level of AR protein [5]. Therefore, RNF6 modulates $\mathrm{ER} \alpha$ and increases its stability in a manner different from ubiquitination.

RNF6 plays a critical role in prostate cancer progression by regulating AR transcriptional activity as a transcription factor because knockdown of RNF6 abolishes dihydrotestosterone-induced and androgenindependent AR activation. Specifically, RNF6-mediated ubiquitination modification is required for AR modulated transcription of a subset cognate target genes including PSA and RLN1 [5]. In breast cancer cells, three signature genes including ER, PR and HER2 determine chemoresistance and prognosis of breast cancers [13]. In the present study, RNF6 is highly associated with ER and PR but not HER2, specifically RNF6 stabilizes ER $\alpha$ and upregulates the expression of Bcl-xL, a key gene in cancer cell survival. Therefore, RNF6 probably regulate the ER $\alpha$ / $\mathrm{Bcl}-\mathrm{xL}$ axle for the pathophysiology of breast cancer in a manner different from its role in prostate cancer and leukemia.

Taken together, the present study demonstrates that RNF6 triggers the ER $\alpha / \mathrm{Bcl}-\mathrm{xL}$ axle thus promoting proliferation, migration and chemoresistance of breast cancer cells. This study adds a novel line of evidence in breast cancer progression and may help in understanding breast cancer therapeutics.

\section{MATERIALS AND METHODS}

\section{Primary breast cancer tissues and adjacent tissues}

Fresh breast cancer tissues and corresponding adjacent normal tissues were collected from 32 female patients between 2013-2014 from the Suzhou Municipal Hospital East Campus, Suzhou, China. Of these cancer tissues, 27 were infitrating ductal carcinomas and 5 were intra-ductal carcinomas based on pathological studies. These tissues were preserved in liquid nitrogen for further studies. In addition, 136 infitrating ductal carcinoma tissues embedded in paraffin from Breast Cancer Bank of Suzhou Municipal Hospital East Campus were subjected to tissue arrays by immunostaining. The patient characteristics are summarized in Table 1. This study was 
conducted in accordance with the Declaration of Helsinki, International Conference on Harmonization Good Clinical Practice, and nationally mandated ethical requirements. The study protocol was reviewed and approved by the ethics committee of Suzhou Municipal Hospital and the written informed consent was obtained from the human subjects.

\section{Cell lines}

Breast cancer cell lines including MCF-7, MDA231, T47D and MDA-453 were originally obtained from American Type Culture Collection (Manassas, VA); Doxorubicin-resistant MCF-7 (MCF-7 $\left.{ }^{\mathrm{R}}\right)$ cells [16] were provided by Dr. Zhiyuan Zhong, Soochow University; HEK293 cells were obtained from Dr. Aaron Schimmer (the University of Toronto, Canada). Breast cancer cells and HEK293 cells were maintained in RPMI-1640 and Dulbecco's high glucose modified Eagle's medium (DMEM) (Hyclone), respectively. All media were supplemented with $10 \%$ fetal calf serum $\left(\right.$ Biowest $^{\circledR}$, Nuaillé, France), $100 \mu \mathrm{g} / \mathrm{ml}$ penicillin, and $100 \mathrm{U} / \mathrm{mL}$ streptomycin.

\section{Reverse transcription-polymerase chain reaction (RT-PCR) and Quantitative real-time polymerase chain reaction (qRT-PCR)}

To determine the mRNA levels of RNF6, RT-PCR and qRT-PCR was performed as described previously [6].

\section{Preparation of RNF6 lentivirus and viral transduction}

The full-length RNF6 gene was amplified by PCR as described previously [6]. The lentivirus-delivered shRNAs against RNF6 (shRNF6) together with the negative control (shNC) were purchased from GeneChem Co, Ltd (Shanghai, China). Lentiviruses were prepared with a standard protocol as manufacturer's instructions as described previously [6].

\section{Cell growth and viability}

MCF-7 cells infected with RNF6 or shRNF6 or scramble lentivirus were cultured for 0 to 5 days at a density of $2 \times 10^{4}$ cells/well in a 24 -well plate. Cell viability was evaluated by MTT assay as described previously [17].

\section{Survival curve analysis}

To estimate the survival period of breast cancer patients in association with RNF6, 136 patients were divided into two groups according to the expression level of RNF6 and the survival rate was calculated according to the Kaplan-Meier estimates described previously [18].
The Kaplan-Meier survival curve was plotted based on the survival rate of each group.

\section{Immunoblotting}

Whole cell lysates were prepared as described previously [19]. Equal amounts $(30 \mu \mathrm{g})$ of total proteins were subjected to sodium dodecylsulfate-polyacrylamide gel electrophoresis (SDS-PAGE) separation, followed by immunoblotting analyses with specific antibodies including antibodies against RNF6 (Thermo Fisher), Flag, Myc, HA (Medical \& Biological Laboratories, Tokyo, Japan), ER $\alpha$, Bcl-xL, Bim-1 (Cell Signaling Technologies., Ltd), or GAPDH (Abgent, Suzhou, China). Anti-mouse immunoglobulin $\mathrm{G}$ (IgG) and anti-rabbit IgG horseradish peroxidase conjugated antibody were purchased from R\&D Systems.

\section{Immunohistochemistry}

Breast cancer tissues were fixed in 10\% neutral buffered formalin before being embedded in paraffin. The tissues were then cut into 6-micron thickness with a microtome. The slides were deparaffinized and rehydrated before antigen retrieval as described previously [20]. All slides were then subject to blocking in $10 \%$ normal serum for 10 minutes, followed by incubation with antibodies against RNF6, ER $\alpha$, PR or HER2, overnight at $4{ }^{\circ} \mathrm{C}$. A biotin-conjugated secondary antibody diluted with Tris-based buffer (TBS) containing 10\% serum and $1 \%$ BSA was applied to incubate for 10 minutes. After rinsing with cold TBS, the slides were then incubated with Streptavidin-peroxidase for 10 minutes before being stained with 3,3'-Diaminobenzidine (Beyotime, Nantong, China). The slides were finally stained with Hematoxylin and eosin before being mounted for microscopy analysis.

\section{Statistics}

Values are expressed as means \pm SD when necessary. The association of RNF6 expression and breast cancer tissues in patients samples were evaluated using the $C h i$ square $\left(\chi^{2}\right)$ analysis. The cell proliferation was evaluated with the student's $t$ test. Survival time was calculated using the Kaplan-Meier method and compared by log-rank test as described previously [18].

\section{ACKNOWLEDGMENTS}

This work was partly supported by National Natural Science Foundation of China (81320108023, 81272632 ), by Natural Science Foundation of Jiangsu Province (BE2014630), by Priority Academic Program Development of Jiangsu Higher Education Institutions (PAPD), and by Jiangsu Key Laboratory for Translational Research and Therapeutics of Neuro-Psycho-Diseases 
(BK2013003). The study was also partly supported by Suzhou Key Medical Center (Szzx201506).

\section{CONFLICTS OF INTEREST}

The authors declare that they have no competing interest.

\section{Authors' contributions}

$\mathrm{YZ}, \mathrm{XX}, \mathrm{SW}, \mathrm{ZZ}, \mathrm{YL}, \mathrm{HK}$, and $\mathrm{BC}$ conducted experiments; $\mathrm{XM}$ and $\mathrm{YZ}$ designed the study, analyzed data and wrote the manuscript.

\section{REFERENCES}

1. McGuire S. World Cancer Report 2014. Geneva, Switzerland: World Health Organization, International Agency for Research on Cancer, WHO Press, 2015. Adv Nutr. 2016; 7:418-419.

2. Shi J, Liang D, Jin J, Wang L, He Y. Female breast cancer burden was increasing during the 40 years in Hebei Province, China: a population-based study. Arch Gynecol Obstet. 2016.

3. Macdonald DH, Lahiri D, Sampath A, Chase A, Sohal J, Cross NC. Cloning and characterization of RNF6, a novel RING finger gene mapping to 13q12. Genomics. 1999; 58:94-97.

4. Lo HS, Hu N, Gere S, Lu N, Su H, Goldstein AM, Taylor PR, Lee MP. Identification of somatic mutations of the RNF6 gene in human esophageal squamous cell carcinoma. Cancer Res. 2002; 62:4191-4193.

5. Xu K, Shimelis H, Linn DE, Jiang R, Yang X, Sun F, Guo Z, Chen H, Li W, Kong X, Melamed J, Fang S, Xiao Z, et al. Regulation of androgen receptor transcriptional activity and specificity by RNF6-induced ubiquitination. Cancer Cell. 2009; 15:270-282.

6. Xu X, Han K, Tang X, Zeng Y, Lin X, Zhao Y, Zhang Z, Cao B, Wu D, Mao X. The Ring Finger Protein RNF6 Induces Leukemia Cell Proliferation as a Direct Target of Pre-B-cell Leukemia Homeobox 1. J Biol Chem. 2016; 291:9617-9628.

7. Holliday DL, Speirs V. Choosing the right cell line for breast cancer research. Breast Cancer Res. 2011; 13:215.

8. Jehn CF, Hemmati P, Lehenbauer-Dehm S, Kummel S, Flath B, Schmid P. Biweekly Pegylated Liposomal Doxorubicin (Caelyx) in Heavily Pretreated Metastatic Breast Cancer: A Phase 2 Study. Clin Breast Cancer. 2016.

9. Li X, Wood TE, Sprangers R, Jansen G, Franke NE, Mao X, Wang X, Zhang Y, Verbrugge SE, Adomat H, Li ZH, Trudel $\mathrm{S}$, Chen $\mathrm{C}$, et al. Effect of noncompetitive proteasome inhibition on bortezomib resistance. J Natl Cancer Inst. 2010; 102:1069-1082.
10. Zhong Y, Wang C, Cheng L, Meng F, Zhong Z, Liu Z. Gold nanorod-cored biodegradable micelles as a robust and remotely controllable doxorubicin release system for potent inhibition of drug-sensitive and -resistant cancer cells. Biomacromolecules. 2013; 14:2411-2419.

11. Pandya V, Glubrecht D, Vos L, Hanson J, Damaraju S, Mackey J, Hugh J, Goping IS. The pro-apoptotic paradox: the $\mathrm{BH} 3$-only protein $\mathrm{Bcl}-2$ interacting killer (Bik) is prognostic for unfavorable outcomes in breast cancer. Oncotarget. 2016; 7:33272-85. doi: 10.18632/ oncotarget.8924.

12. Vaillant F, Merino D, Lee L, Breslin K, Pal B, Ritchie ME, Smyth GK, Christie M, Phillipson LJ, Burns CJ, Mann GB, Visvader JE, Lindeman GJ. Targeting BCL-2 with the BH3 mimetic ABT-199 in estrogen receptor-positive breast cancer. Cancer Cell. 2013; 24:120-129.

13. Yeung C, Hilton J, Clemons M, Mazzarello S, Hutton B, Haggar F, Addison CL, Kuchuk I, Zhu X, Gelmon K, Arnaout A. Estrogen, progesterone, and HER2/neu receptor discordance between primary and metastatic breast tumours-a review. Cancer Metastasis Rev. 2016.

14. Collignon J, Lousberg L, Schroeder H, Jerusalem G. Triplenegative breast cancer: treatment challenges and solutions. Breast Cancer (Dove Med Press). 2016; 8:93-107.

15. Dent R, Trudeau M, Pritchard KI, Hanna WM, Kahn HK, Sawka CA, Lickley LA, Rawlinson E, Sun P, Narod SA. Triple-negative breast cancer: clinical features and patterns of recurrence. Clin Cancer Res. 2007; 13:4429-4434.

16. Zhong Y, Zhang J, Cheng R, Deng C, Meng F, Xie F, Zhong $Z$. Reversibly crosslinked hyaluronic acid nanoparticles for active targeting and intelligent delivery of doxorubicin to drug resistant CD44+ human breast tumor xenografts. J Control Release. 2015; 205:144-154.

17. Han K, Xu X, Xu Z, Chen G, Zeng Y, Zhang Z, Cao B, Kong Y, Tang X, Mao X. SC06, a novel small molecule compound, displays preclinical activity against multiple myeloma by disrupting the mTOR signaling pathway. Sci Rep. 2015; 5:12809.

18. Goel MK, Khanna P, Kishore J. Understanding survival analysis: Kaplan-Meier estimate. Int J Ayurveda Res. 2010; 1:274-278.

19. Mao X, Hou T, Cao B, Wang W, Li Z, Chen S, Fei M, Hurren R, Gronda M, Wu D, Trudel S, Schimmer AD. The tricyclic antidepressant amitriptyline inhibits D-cyclin transactivation and induces myeloma cell apoptosis by inhibiting histone deacetylases: in vitro and in silico evidence. Mol Pharmacol. 2011; 79:672-680.

20. Hsu SM, Raine L, Fanger H. Use of avidin-biotinperoxidase complex $(\mathrm{ABC})$ in immunoperoxidase techniques: a comparison between $\mathrm{ABC}$ and unlabeled antibody (PAP) procedures. J Histochem Cytochem. 1981; 29:577-580. 